\title{
Quality indicators for mental health in primary care: how far have we got?
}

\section{J Sharp}

\section{The development of valid indicators to assess the quality of care for mental health is a challenge for primary care organisations.}

Q uality of care-be it at the level of the institution or the individual healthcare professional-is at the core of the modernisation of the NHS, ${ }^{1}$ but nowhere is it more difficult to measure and assess than in the area of mental health. Unlike diabetes mellitus and, to some extent, coronary artery disease where there is good agreement on the absolute threshold for making the diagnosis and increasing consensus as to what processes constitute high quality care (e.g. retinal screening, use of aspirin), the definite diagnosis of a mental health problem and agreement on its optimum management is much less clear. This is particularly so for the large burden of mainly undifferentiated mental illness that presents in primary care. Although undifferentiated, it should not be assumed that the severity and accompanying functional impairment are not insubstantial. We are not, as some might assert, dealing with the "worried well".

In secondary care, psychiatrists are better able to agree on the diagnosis, partly because they are more used to using internationally agreed classification systems. In addition, more research has been undertaken to examine both the process and content of care for patients with long term enduring mental illness-for example, integrated care pathways and a stepped approach to medication. With harder outcomes available for measurement, such as the number of hospital admissions, quality of care is both easier to define and to measure. However, in primary care where the various stakeholders are often unable to agree whether or not a mental illness is present, how severe it is, and the degree of impairment, consensus on management is much more difficult and developing quality indicators is an uphill struggle.

For many people the concept of quality indicators is a new one. If we agree that the range of professional practice with regard to mental illness in primary care is unacceptable and thus quality is often suboptimal, we must take steps to measure it objectively. In their recent publication Marshall et al ${ }^{2}$ state that:
"Quality indicators are specific and measurable elements of practice that can be used to assess the quality of care. They are usually derived from retrospective reviews of medical records or routine information sources. Some authorities differentiate 'quality' from 'activity' or 'performance' indicators. The important issue is that a good quality indicator should define care that is attributable and within the control of the person who is delivering the care."

It is the final sentence in this definition that is key. Quality is a complex construct that is likely to have a different meaning for patients, professionals, and managers. However, where objective evidence of variation exists-for example, in access, in effectiveness or in efficiency-patients and professionals are likely to agree that some process is required by which absolute and relative indices of quality can be measured. The task is to develop valid (quality) indicators for mental health care for which individual health professionals can take responsibility. What is particularly difficult in mental health is agreement on the gold standard.

The approach to developing quality indicators taken by Shield $e a^{3}$ in their paper in this issue of QSHC incorporated the views of 11 different groups of stakeholders. After two rounds of a Delphi process, only one quarter of more than 300 indicators-derived from a variety of sources including published guidelines, focus groups, and the National Service Framework (NSF) for Mental Health ${ }^{4}$ were rated valid by all panels. Furthermore, whereas carers rated $91 \%$ of indicators as valid, GPs rated only $41 \%$ valid. Who has primacy here? The indicators on which there was consensus were grouped into 21 aspects of care, 11 relating to general practices and 10 to health authorities or primary care groups/trusts. However, if we agree with the definition of Marshall et $a l^{2}$ that, for an indicator to be valid, it must be "attributable and within the control of the person who is delivering the care", very few of the quality indicators derived have the health professional/patient interface at their core. Furthermore, many of the indicators could be described as generic in that they apply equally to a patient with diabetes or coronary heart disease-for example, being able to make an appointment to see a GP within 48 hours or being provided with appropriate information about one's condition.

This leads us back to the difficulty of developing valid indicators specifically for mental health. The evidence base on which to make unequivocal statements in primary care mental health is still somewhat flimsy, but this should not stop us from making a start. The idea of measuring quality in health care is not new. Over 20 years ago Donabedian and coworkers ${ }^{5}$ proposed a multidimensional matrix of structure, process, and outcome to help define and then measure the quality of health care. How can we use this matrix in the field of primary care mental health? A starting point might be the common mental disorders-depression and anxiety. Thus, under "structure" one might decide that depressed patients who perceive the need could book a longer appointment with the doctor; under "process" the primary care team might agree a formulary for antidepressant prescribing; and the "outcomes" might include the number of patients who complete a full course of antidepressants. Each of these constructs can be translated into a quality indicator for which an individual has responsibility.

Mental illness is a major burden for the NHS. The NSF for mental health ${ }^{4}$ is but one of a number of initiatives that aim to improve quality of care. Whether this "top down" approach can drive quality at the interface between health professionals and patients is unclear. The Sainsbury Centre for Mental Health takes the quality agenda somewhat nearer to the service users and suggests a set of more focused standards for primary care organisations, some of which primary care teams should find easier to develop into quality indicators. ${ }^{6}$ The challenge will then be to undertake the necessary research using rigorous methodology, the results of which will allow us to make evidence based changes in the search for further quality improvement. $^{7}$

Qual Saf Health Care 2003;12:85-86

\section{Author's affiliation}

D J Sharp, Professor of Primary Health Care, University of Bristol, Cotham House, Bristol BS6 6JL, UK; debbie.sharp@bristol.ac.uk

\section{REFERENCES}

1 Department of Health. The NHS plan: a plan for investment, a plan for reform. London: Department of Health, 2000.

2 Marshall M, Campbell C, Hacker J, et al, eds. Quality indicators for general practice. London: Royal Society of Medicine, 2002. 
3 Shield TL, Campbell SM, Rogers A, et al. Quality indicators for primary care menta health services. Qual Saf Health Care 2003;12:100-6

4 Department of Health. The national service framework for mental health. London: Department of Health, 1999.
5 Donabedian A, Wheeler JRC, Wyszewlanski . Quality, cost and health: an integrative model. Med Care 1982;20:975-92.

6 Sainsbury Centre for Mental Health.

Setting the standard: the new agenda for primary care organisations commissioning mental health services. London: Sainsbury

Centre for Mental Health, 2001

7 Grol R, Baker R, Moss F. Quality

improvement research: understanding the

science of change in health care. Qual Saf

Health Care 2002;11:110-11.

\section{A call for internet pharmacies to comply with quality standards}

\section{Anderson}

\section{Internet pharmacies need to consider the quality of their services and to comply with the standards set for them by national pharmaceutical associations.}

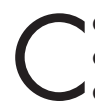
onsumers are increasingly being encouraged to adopt a philosophy of self-care; the internet allows people to obtain advice about treatment and medicines at their own convenience. Studies indicate that there are many inaccuracies in health information on the internet, and a widely varying quality of information with patients in essence taking a "lucky dip" as to which site they choose to view and how good the quality of information is. There may be even greater problems if the sites offer to supply medicines as in the case of many e-pharmacies. It appears that very few e-pharmacies provide the facility for customers to have a meaningful consultation with a pharmacist. A number of national pharmaceutical organisations have developed ethical guidance regarding the operation of e-pharmacies, but this, it appears, is rarely adhered to.

In this issue of QSHC Bessell et al report a study which evaluated the quality of information published on global websites selling medicines using the DISCERN tool and examined what happened when a "pseudo customer" ordered a non-prescription medicine (pseudoephedrine) and a complementary medicine (St John's wort) online; the customer was also taking fluoxetine (Prozac).

The methodology used is a very useful one for evaluating e-pharmacies. Internet pharmacies-like community pharmacies in general-are open to scrutiny by pseudo customers or mystery shoppers as people can ask for advice and purchase over the counter medicines with no need for registration with the pharmacy. It was good practice to send the results to the e-pharmacies for comment.
In the study by Bessell et al 63 out of 104 websites investigated provided some health information but, overall, the quality of that information as judged by the DISCERN tool was poor. What is particularly sobering is that only three websites provided adequate advice to consumers to avoid a potential drug interaction. Only 27 of the sites actually sold medicines and, of those, only three asked the customer for further information about a potential drug interaction by contacting the customer by telephone and advising them to seek medical advice. One pharmacy sent out of date stock and another sent a substitute brand without checking if it was all right to do so.

\section{". . . it is probably not safe to surf and self-medicate"}

Interaction via a computer terminal allows customers to be completely anonymous and, as such, there can be no proper checks on the customer's state of health, other medications, or even age. However, people may be more willing to describe their symptoms over a computer terminal than face-to-face with their doctor or pharmacist, particularly if they find the complaint embarrassing. Any advice provided, particularly concerning drugs, could subsequently prove damaging to the patient if all relevant information is not elicited, so this presents a danger-both to the public and to the reputation of the pharmacy profession for providing high quality impartial advice.

The main attribute of the internet is also its main problem-namely, its global nature. No one body controls the whole of the internet so no one body can oversee and ensure the quality and accuracy of its information. It may ultimately come down to the individual user to choose which health based website to trust and use. There is much debate about evaluating websites containing health information and about who should perform that evaluation. ${ }^{23}$ The New Zealand Pharmaceutical Society (www.psnz.org.nz) has set up an accreditation scheme for internet pharmacies. Accreditation provides reassurance that the internet pharmacy is fully licensed, observes quality pharmacy practices, complies with patient rights to privacy and confidentiality, complies with ethical codes and legislative requirements for the advertising of medicines, provides factual and understandable information about all medicines advertised, and provides opportunity for meaningful consultation between patient and pharmacist.

As Bessell et al conclude, it is probably not safe to surf and self-medicate. Consumers who self-select medicines from websites have insufficient access to information and advice to make informed decisions about the safe and appropriate use of medicines. There is a need to develop more customer focused services. Internet pharmacies need to consider the quality of their services and to start to comply with the standards set for them by national pharmaceutical associations.

Qual Saf Health Care 2003;12:86

Author's affiliation

C W Anderson, Reader in Pharmacy Practice and Social Pharmacy, The Pharmacy School, University of Nottingham, Nottingham NG7 2RD, UK

claire.anderson@nottingham.ac.uk

\section{REFERENCES}

1 Bessell TL, Anderson JN, Silagy CA, et al Surfing, self-medicating and safety: buying non-prescription and complementary medicines via the internet. Qual Saf Health Care 2002; 11:88-92.

2 Delamothe T. Quality of websites: kitemarking the west wind. BM 2000;321:843-4.

3 Eysenbach G, Yihune G, Lampe K, et al. Website labels are analogous to food labels BM 2001;322:794. 
Patient involvement

\section{Clinicians' and patients' roles in patient involvement}

\section{N Britten}

\section{Development of the OPTION instrument will enable researchers to measure the extent to which clinicians involve patients in decisions within consultations.}

$\mathrm{T}$ he involvement of patients in health care is now a matter of government policy, at least in the UK. Partly as a result of the scandal surrounding paediatric heart surgery in Bristol and the ensuing enquiry, ${ }^{1}$ public and patient involvement is now firmly on the agenda. To researchers who have been advocating patient centredness and shared decision making for years, if not decades, this is a welcome initiative. However, neither policy makers nor researchers have made much progress in working out how patient involvement is to be measured. Involvement can occur at many levels-from citizens' juries to patient participation groups-but perhaps the most important arena for many people is the face to face consultation with a clinician.

The value of the carefully conducted work by Elwyn et al published in this issue of QSHC is, firstly, that it established-by means of a systematic review-that there was no existing measure of the extent to which clinicians involve patients in decisions within consultations and, secondly, that it has succeeded in developing such a measure. The resulting instrument, OPTION, will enable researchers to investigate the links between process in the consultation and subsequent outcomes. The literature is full of theoretical and abstract models which in many cases have not been turned into research tools. ${ }^{3}$ The
OPTION instrument will allow the model of shared decision making to be evaluated in terms of its occurrence in actual practice and its consequences for both patient and professionally defined outcomes. It will allow researchers to explore whether or not shared decision making leads to better clinical decisions, the achievement of patients' goals, higher levels of adherence, and better health outcomes. Another important question which can now be addressed is the extent to which patients from different social backgrounds have different expectations and/or are treated differently. ${ }^{4}$ The results of the study by Elwyn et al showed that the doctors in their sample rarely established patients' preferred level of involvement in decision making. Thus, if doctors are treating less educated patients differently from those with more education, or if they are less likely to involve patients with fewer resources in decisions about their care, such differences may reflect doctors' assumptions and not the actual preferences of these patients. Such differential involvement, if it exists, may help to explain the inequalities in access to and take up of health care. The instrument developed by Elwyn et al will allow these and other possibilities to be explored.

However, as the authors acknowledge, OPTION does not measure the patient's contribution or the actual interaction between patient and professional. To that extent it is a professional or doctor centred instrument. The "skills framework" identified refers to the skills of the professional, not of the patient. It can be argued that the onus is on professionals to change their behaviour as they spend more time in consultations than patients usually do. In fact, in any interaction both (or all) participants are usually contributing in some form or other. The level of variation within practitioners in the study by Elwyn et al, which exceeded variation between practitioners, suggests that patients' behaviour might explain at least some of what practitioners were doing. You cannot understand fully what clinicians are doing if you do not also look at what patients are doing, which is to say that health care consultations are co-constructed..$^{5}$ As well as establishing patients' preferences for involvement in decision making, researchers need to examine what patients of all backgrounds are actually doing in the consultation already, and the ways in which current patterns of involvement or noninvolvement are co-produced by clinicians and patients.

Qual Saf Health Care 2003;12:87

\section{Author's affiliation}

N Britten, Professor of Applied Health Care Research, Institute of Clinical Education, Peninsula Medical School, St Luke's Campus, Exeter EX1 2LU, UK; nicky.britten@pms.ac.uk

\section{REFERENCES}

1 Secretary of State for Health. Learning from Bristol: the report of the Public Inquiry into children's heart surgery at the Bristol Royal Infirmary 1984-95. CM5207(II). London: Department of Health, 2001

2 Elwyn G, Edwards A, Wensing $M$, et al Shared decision making: developing the OPTION scale for measuring patient involvement. Qual Saf Health Care 2003;12:93-9

3 Charles C, Gafni A, Whelan T. Decision-making in the physician-patient encounter: revisiting the shared treatment decision-making model. Soc Sci Med 1999;49:651-61.

4 Dieppe P, Horne R. Soundbites and patient centred care. BM 2002;325:605

5 Gafaranga J, Britten N. "Fire away": the opening sequence in general practice consultations. Fam Pract 2003 (in press). 